\title{
LA PLANIFICACIÓN POR COMPETENCIAS EN LA REFORMA DE BOLONIA DE LA EDUCACIÓN SUPERIOR: UN ANÁLISIS CRÍTICO
}

\section{Antonio Bolívar}

\section{RESUMEN}

Este trabajo analiza la dimensión curricular y didáctica, más que la estructural, de la reforma de Bolonia. Se describen las principales líneas y orientaciones de la planificación del currículum y la enseñanza según el Proceso de Convergencia de Bolonia, pensando que puede ofrecer una oportunidad institucional para mejorar la docencia en la Universidad, a condición de no limitarse a cambios estructurales, para lo que debe incidir en mudar la propia mentalidad y cultura establecida. Más particularmente, se realiza un análisis crítico del papel que juegan las competencias en el proceso de planificación, tanto en el modelo adoptado (Proyecto Tuning), como por la subordinación al perfil profesional y su vinculación con el mercado laboral. Desde un abordaje crítico se defiende que, si bien la Universidad debe preparar para el ejercicio profesional, ello no puede suponer desdeñar otras dimensiones de la Educación Superior.

\section{PALABRAS CLAVE}

Processo de Bolonia; Planificación educacional; Competencias; Perfil profesional; Proyecto tuning

\section{A PLANIFICAÇÃO POR COMPETÊNCIAS A REFORMA DE BOLONHA DA EDUCAÇÃO SUPERIOR: UMA ANÁLISE CRÍTICA}

\begin{abstract}
RESUMO
Este trabalho analisa a dimensão curricular e didática, mais do que a estrutural, da reforma de Bolonha. Descrevem-se as principais linhas e orientações da planificação do currículo e do conhecimento segundo o Processo de Convergência de Bolonha, pensando que se pode oferecer uma oportunidade institucional para melhorar a docência da Universidade. Para que isto aconteça, a condição é de que o processo não se limite a câmbios estruturais, mas sim o que deve incidir em mudar a própria mentalidade e cultura estabelecida. Mais particularmente, se realiza uma analise critica do papel que se julgam as competências do processo de planificação, tanto no modelo adaptado (Prometo Tuning), como pela subordinação do perfil profissional, e sua vinculação com o Mercado de Trabalho. Desde uma abordagem critica se defende que a Universidade deve preparar para o exercício profissional, e isto não pode supor desdenhar outras dimensões da Educação Superior.
\end{abstract}

PALAVRAS-CHAVE

Reforma de Bolonha; Planificação educacional; Competências; Perfil profissional; Projeto tuning

(C) ETD - Educação Temática Digital , Campinas, v.9, n. esp., p.68-94, dez. 2007 - ISSN: 1676-2592. 


\section{INTRODUCCIÓN}

La construcción del Espacio Europeo de Educación Superior ("European Higher Education Area” o “Bologna Process”), como consecuencia de la Declaración de Bolonia, se presenta como una oportunidad histórica para armonizar la Educación Superior europea, de modo que posibilite una movilidad de estudiantes y profesionales, al tiempo que para una renovación didáctica de la propia enseñanza universitaria. La implementación por cada país de los sucesivos acuerdos europeos está planteando nuevos desafíos a la Universidad, incidiendo en el currículum, docencia y trabajo de los estudiantes y del profesorado ${ }^{1}$. Estamos ya cercanos al año 2010 en que, según los compromisos adquiridos, toda la nueva estructura debe estar puesta en marcha. En España, por los sucesivos cambios de Gobierno y ministros, así como de legislación, se han dado avances y paralizaciones, según dichos cambios ministeriales, encontrándonos un tanto retrasados, por lo que en los próximos dos años habrá que hacer el diseño de todas las nuevas titulaciones universitarias, adecuar los postgrados e iniciar su implementación de modo generalizado en todas las Universidades.

Los objetivos estratégicos de la creación del EEES (Espacio Europeo de Educación Superior), según se enuncian en la Declaración de Bolonia y han sido precisados posteriormente, se centran en: construir un sistema fácilmente comprensible y comparable de titulaciones que permitan fomentar el acceso al mercado laboral e incrementar la competitividad del sistema universitario europeo para que se convierta en un destino atractivo para los estudiantes y profesores de otras regiones del mundo; establecer un sistema basado fundamentalmente en dos ciclos principales (Grado/Postgrado); adoptar un sistema de créditos compatibles que promocione la movilidad de estudiantes y profesores; impulsar la cooperación europea para garantizar la calidad de la Educación Superior a través del desarrollo de redes, proyectos conjuntos, agencias u organismos específicos de soporte, etc., para definir criterios y metodologías comparables.

\footnotetext{
${ }^{1}$ Ver sobre el tema, entre otros, EURYDICE. Organización de la estructura de la Educación Superior en Europa 2004/2005: Tendencias nacionales en el Marco del Proceso de Bolonia. Madrid : Ministerio de Educación y Ciencia, Subdirección General de Información y Publicaciones, 2007, 234 p. Disponible en Internet en http://www.mec.es/cide También para ver la situación en los dos años siguientes, EURYDICE. Focus on the structure of Higher Education in Europe 2006/07: National trends in the Bologna Process. Brussels: Eurydice, European Unit, 2007, 349 p. Disponible en http://www.eurydice.org . Por lo demás la propia página del Proceso de Bolonia en http://www.bologna-bergen2005.no/ , ahora también en la nueva web ("Bolonia Benelux”) con motivo de la próxima Conferencia ministerial en Lovaina en 2009: http://www.ond.vlaanderen.be/hogeronderwijs/bologna/ . Por lo demás, en tercer lugar, la Asociación Europea de Universidades ha publicado sucesivos Informes (Trends, I,II, III, IV y V) en que informa del desarrollo del proceso de Bolonia en los distintos países. Los documentos puede consultarse en http://www.eua.be/eua/index.jsp , acceso en: 25 jun. 2007.
} 
Los elementos principales, pues, que configuran el nuevo escenario del EEES son: establecimiento generalizado del llamado crédito europeo (ECTS en siglas de acuerdo con la denominación oficial: European Credit Transfer System), currículos estructurados en dos niveles (Grado/Postgrado, este último subdivido en Máster y Doctorado), acreditación para asegurar la calidad y transparencia mediante el suplemento europeo al título. Todo ello subordinado al objetivo más general de promover la movilidad, el reconocimiento mutuo de los estudios entre instituciones y países y la propia competitividad. El peligro que amenaza, como todo proceso de cambio, es quedarse a nivel formal de estructuras, sin penetrar en la “cultura”, modos heredados de hacer o mentalidad, tanto del profesorado y del alumnado, sin llegar a alterar cualitativamente los modos mismos de enseñar y aprender (GOÑI, 2005; COLÁS; DE PABLOS, 2005; PEREYRA; LUZÓN; SEVILLA, 2006).

La adaptación al EEES o Proceso de Bolonia ha ido vinculada en España, en el nuevo diseño y estructura de las titulaciones universitarias, al discurso de las “competencias”. De modo acrítico, se ha adoptado, en un primer momento, como modelo base de diseño curricular en la reforma de las titulaciones, el Proyecto Tuning Educational Structures in Europe (GONZÁLEZ; WAGENAAR, 2003), que sitúa las competencias en el centro de la delimitación de los denominados "perfiles profesionales”; así como en el llamado nuevo paradigma educativo de “educación centrada en el aprendizaje del estudiante”. Las competencias se convierten en los logros del aprendizaje, en lugar de la adquisición de conocimientos, afectando a los objetivos, al papel del profesor, a las actividades de enseñanza y a la propia evaluación. En España, la Agencia Nacional de Evaluación de la Calidad y Acreditación (ANECA), encargada de orientar y supervisar el proceso, desde el primer momento, adoptó (e impone en sus respectivas convocatorias) un modelo único de Diseño basado en las competencias, de acuerdo con la formulación que había realizado Tuning. Así, en las tres convocatorias de la ANECA de 2003 y 2004 para el diseño de Planes de Estudios y Títulos de Grado se exigía adecuarse a unas líneas directrices y orientaciones ${ }^{2}$ en este sentido.

\footnotetext{
${ }^{2}$ Sobre el asunto se puede ver en la web de la ANECA el apartado: "El Programa de Convergencia de la ANECA (Agencia Nacional de Evaluación de la Calidad y Acreditación)”, disponible en Internet en http://www.aneca.es/activin/activin_conver.asp . Un resumen y resultados del Programa de la ANECA sobre el diseño de los Planes de Estudios y Titulos se puede ver en la publicación reciente El Programa de Convergencia Europea de ANECA (2003-2006). Disponible en la propia web: http://www.aneca.es/active/active_ense_pei_0506.asp Por último uno de los frutos de dicha tarea planficadora, que pueden servir de casos ejemplares, han sido los llamandos “Libros Blanco" de cada titulación universitaria. Disponibles en Internet en http://www.aneca.es/activin/activin_conver_LLBB.asp
} 
Por lo demás este asunto ha pasado a ser ya paneuropeo, constituyéndose también -por las expectativas que ha suscitado- en un proceso a adecuar en América Latina y el Caribe. Además de las reuniones oficiales para poner en marcha un proceso similar, se ha desarrollado el proyecto Alfa Tuning América Latina, apoyado por la Comisión Europea y en el que participan 19 países iberoamericanos (entre ellos Brasil), que pretende contribuir al desarrollo de titulaciones fácilmente comparables y comprensibles de una forma articulada en toda América Latina, buscando puntos de referencia comunes y centrándose en las competencias y destrezas. En este sentido, el proyecto tiene, al igual que el europeo, cuatro grandes líneas: definición de las competencias, enfoques de enseñanza y aprendizaje, créditos académicos, y calidad de los programas ${ }^{3}$. Además, se ha iniciado un fructífero intercambio entre las instituciones de Educación Superior europeas e iberoamericanas para el incremento de la calidad, efectividad y transparencia.

En este trabajo nos centramos en la dimensión curricular y didáctica, más que en la estructural, del proceso de Bolonia; por una parte, describimos las líneas directrices del nuevo Diseño de Guías de las Titulaciones según el Proceso de Convergencia de Bolonia, pensando que el proceso de convergencia puede ofrecer una oportunidad institucional para mejorar la docencia en la Universidad, a condición de no limitarse a cambios estructurales, afectando a la propia mentalidad y cultura establecida. Más particularmente, hacemos un análisis crítico del papel que juegan las competencias en el proceso de planificación.

\section{DISEÑO DE PLANES DE ESTUDIO DE TITULACIONES: CENTRADOS EN APRENDIZAJE Y COMPETENCIAS}

Toda una larga tradición desde Tyler, calificada de enfoque técnico-administrativista, ha puesto en la planificación o diseño del currículum la base de la mejora de la enseñanza. En el propio ámbito universitario, la Red Europea para garantizar la Calidad en la Educación Superior (ENQA, 2005) señala que la clave de la calidad depende de una mejora técnica de la planificación de la docencia, situando como eje el aprendizaje de los estudiantes. Si bien no vamos a restar relevancia a la planificación, también en muchos casos la tarea del diseño se ha quedado en mera formalidad, al servicio de requerimientos administrativos. Más allá de una lógica propia de una racionalidad burocrática, interesada en cumplir con determinados

\footnotetext{
${ }^{3}$ Cfr. BENEITONE, Pablo; ESQUETINI, César; GONZÁLEZ, Julia et al. (eds.). Informe Final del Proyecto Tuning América Latina: Reflexiones y perspectivas de la Educación Superior en América Latina. Bilbao: Publicaciones de la Universidad de Deusto. 2007. Disponible en la página web del Proyecto Tuning dedicada a América Latina http://tuning.unideusto.org/tuningal/ acceso en: 25 jun. 2007
} 
“formatos”, se ha de poner el acento en el propio proceso de trabajo conjunto y la revisión continuada de la planificación realizada en función de su incidencia en el desarrollo práctico. Tal y como está funcionando en España el peligro es real, al exigir y hacer creer al profesorado que el asunto se juega primariamente en rellenar bien determinados formatos.

El Diseño de una Titulación es un proceso de reflexión y toma de decisiones sobre los objetivos que se quieren lograr, los contenidos (asignaturas y programas) que pueden ser más adecuados, así como los procesos de enseñanza-aprendizaje que se van a desarrollar. Se trata de prever la articulación de procesos de la práctica futura, analizando los elementos que intervienen en función de las competencias necesarias para perfil de profesionales que queremos que forme el sistema universitario. A su vez, la propuesta docente ha de ser justificada de forma que adquiera sentido en un contexto profesional y disciplinar determinado. Formalizar el plan tiene como función proporcionar una visión de conjunto y es un cierto compromiso público de la formación que se pretende proporcionar. La planificación de los programas de las asignaturas se ha identificado con la determinación de contenidos que, normalmente, se desarrollarán en clase, con metodología expositiva y de manera presencial. El nuevo enfoque que propugna el proceso de Bolonia, en especial con una formación basada en competencias y centrada en el aprendizaje, hace -como dice Concepción Yániz (2006, p. 20)- que "la planificación tenga como referencia el aprendizaje deseado y organice los elementos necesarios para adquirir las competencias que conforman un perfil previamente establecido".

Elaborar el diseño curricular de una titulación suele conllevar las siguientes tareas: seleccionar las competencias a desarrollar; analizar cada competencia e identificar los componentes, habilidades, actitudes y conocimientos, seleccionando los que deban ser trabajados en cada módulo; identificar las asignaturas y otras intervenciones expresamente diseñadas para tal finalidad; formular resultados de aprendizaje que permitan identificar la concreción de las competencias que se les propone desarrollar a los alumnos; planificar el desarrollo de los objetivos eligiendo estrategias de aprendizaje y enseñanza, y diseñando el sistema de evaluación; distribuir el tiempo de trabajo de los estudiantes, según el número de ECTS y horas correspondientes, entre los diferentes módulos, para lo que es fundamental valorar la importancia relativa dentro del proyecto, de las competencias que se están trabajando en cada periodo para que esta distribución sea coherente con el mismo (YÁNIZ, 2006, p. 24-25). 
La formación universitaria, de acuerdo con dicho proceso de reforma de Bolonia, deberá centrarse en la adquisición de competencias por parte de los estudiantes. Por tanto, se pone el énfasis en los resultados del aprendizaje, en aquello que los alumnos son capaces de hacer al término del proceso educativo y en los procedimientos que le permitirán continuar aprendiendo de forma autónoma a lo largo de su vida. Como discutiremos posteriormente, la construcción de un plan de estudios basado en un enfoque de competencias, como señala Diaz Barriga (2006, p. 29), permite elaborar una visión integrada de la formación del profesional en la Educación Superior, al partir de una delimitación de competencias complejas o integradoras; pero, por otra parte, en contrapartida, "su grado de generalidad dificulta su carácter orientador para la toma de decisiones puntuales en diversos tramos de los planes de estudio”.

\subsection{UNA PLANIFICACIÓN CENTRADA EN EL APRENDIZAJE}

La clave de la reforma de la Educación Superior no está primariamente en acomodar los planes de estudio a la nueva estructura, sino en un cambio de orientación o mentalidad. Tanto el crédito europeo como el enfoque de competencias (COMISIÓN EUROPEA, 2003; COROMINAS, 2001; PERRENOUD, 2006; RUÉ, 2007) mudan el paradigma a la hora de plantear los objetivos: de un enfoque centrado en la enseñanza que hace el profesorado se pasa a uno centrado en el aprendizaje de los estudiantes ("The shift from teaching to learning”). El paradigma dominante pone el énfasis en la adquisición y transmisión de conocimiento, centrándose en la enseñanza del profesor y, por eso, la unidad de medida es el trabajo del profesor. En general, la metodología habitual anterior pivota sobre la presencialidad (clases magistrales). Hay un papel de dependencia del alumno y pasividad en el proceso de enseñanza, que se pretende mudar hacia una mayor iniciativa, autonomía acompañada y supervisada, actitud más emprendedora y una mayor cota de responsabilidad de su propio aprendizaje.

Los elementos, que suponen un rediseño de la actividad docente, incluyen pues: una educación centrada en el estudiante, nuevos roles del profesorado y alumnado, mayor determinación en los objetivos, y que las actividades educativas organicen el aprendizaje en torno a la adquisición de competencias. El profesor adquiere el papel de acompañante, de supervisor y guía del aprendizaje, para alcanzar objetivos y competencias previamente definidas. Sin dejar de ser transmisor de conocimiento, éste papel se dirige a mayores niveles de tutoría, de apoyo y motivación para adquirir el conocimiento y habilidades, a la comprensión y capacidad para aplicar ese conocimiento, a la selección crítica de materiales y 
fuentes, organización de situaciones de aprendizaje, etc., con relación al perfil que se desea obtener. Esto suele exigir un menor número de estudiantes para una adecuada tutorización

El nuevo modelo (más cercano al nórdico o anglosajón) prioriza el “aprender a aprender”, donde el conocimiento incluido en las distintas materias de la titulación tiene por objetivo establecer los fundamentos básicos, las competencias o habilidades y las actitudes necesarias para el ejercicio profesional. El proceso, contrariamente al vigente, se centra en el estudiante que aprende y, por eso, la unidad de medida es el trabajo del estudiante (eso es el crédito europeo). Los planes de estudio, pues, a la larga derivan en planes de trabajo de los estudiantes. A su vez, la formación inicial debe complementarse con un proceso de aprendizaje a lo largo de la vida.

\subsection{LAS COMPETENCIAS (GENERALES Y ESPECÍFICAS) EN EL DISEÑO DE LA TITULACIÓN}

El proyecto Tuning Educational Structures in Europe ${ }^{4}$, que se propone la sintonización y armonización de estudios superiores entre países europeos, ha servido de modelo-base para el diseño de las titulaciones en el proceso de convergencia de Bolonia. Este proyecto propone que los créditos ECTS deberían ser formulados en términos de competencias para determinar los logros en el aprendizaje, al tiempo que las competencias pueden servir para expresar la comparabilidad y transparencia entre titulaciones (GONZÁLEZ; WAGENAAR, 2005). De acuerdo con los “perfiles profesionales” se determinan las competencias vinculadas y, a su vez, la selección de conocimientos y contenidos se determinan en función de las competencias (objetivos) de cada perfil profesional. Los objetivos, a nivel general o específico, deben expresarse en términos de competencias que capacitan para un determinado ejercicio profesional (en el Primer Ciclo) o para la especialización e investigación (Segundo Ciclo o Postgrado).

De acuerdo con los perfiles académicos y profesionales, se argumenta que las competencias representan un factor importante que puede guiar la selección de los conocimientos más apropiados para cada objetivo particular. Las competencias están formadas por una combinación dinámica de cualidades -con respecto al conocimiento, a su

\footnotetext{
${ }^{4}$ Cfr. GONZÁLEZ, Julia; WAGENAAR, Robert (2003). Igualmente, más breve, el artículo de los mismos autores: Quality and European Programme Design in Higher Education. European Journal of Education, v. 38, n. 3, p. 241-251, 2003. Más recientemente (2006) se ha publicado el informe final Tuning fase 2 (Universities' contribution to the Bologna Process). La web del Proyecto se puede ver la Universidad de Deusto: http://www.unideusto.org/tuning/ subdividida en Europa y América Latina.
} 
aplicación, a las actitudes y responsabilidades- y se constituyen en los objetivos educativos de un programa de estudios o, dicho de otra manera, en aquello que los alumnos deberán ser capaces de hacer al final de un proceso educativo. Además, permiten comparar la preparación de un alumno más fácilmente que si se hace con los estudios recibidos (éstos pueden nominalmente diferir y, sin embargo, pretender competencias similares).

Los objetivos educativos previstos para el Grado y para el Postgrado deben estar claramente diferenciados. Mientras el primero debe tener una orientación claramente profesionalizadora, el segundo se dirige a la especialización. En la práctica podemos distinguir dos tipos de objetivos educativos, que pueden ser definidos en términos de competencias:

- Competencias generales (transferibles y comunes a cualquier titulación), subdivididas en competencias instrumentales, interpersonales y sistémicas.

- Competencias específicas ligadas a las áreas de estudio (conocimiento teórico, práctico y/o experimental y habilidades específicas del área)

Las competencias generales se refieren a cosas tales como la capacidad de análisis y síntesis, la cultura general, la capacidad para el trabajo independiente, la colaboración y la comunicación, la capacidad para el liderazgo, las habilidades de organización y planificación. En otras palabras, se entienden como capacidades y destrezas que pueden ser utilizadas en muchas situaciones, no sólo en aquellas relacionadas con el área de estudio concreta. Estas competencias, en su mayor parte, se desarrollan utilizando métodos y formas de enseñanzaaprendizaje adecuadas. Además de estas competencias generales, cada titulación intentará potenciar competencias específicas, ligadas al área de estudios (habilidades y conocimientos). Las habilidades relacionadas con el área de estudio hacen referencia a los métodos y técnicas propias y pertinentes del área; por ejemplo, análisis de manuscritos antiguos, análisis químico, técnicas de muestreo, etc., según el área de que se trate.

\section{PLANIFICAR EL CURRÍCULUM UNIVERSITARIO: ELEMENTOS CLAVE}

Una planificación supone prever cómo se quiere que los procesos formativos ocurran en la práctica. Es siempre una actividad intencional, con propósitos definidos, en la que se anticipa un plan que oriente los modos de actuación, disponiendo y conjugando los distintos elementos de la enseñanza. Existen diversas formas de planificar, aún cuando hay un 
consenso en que debe dar respuesta al menos a las siguientes cuestiones (ESTEBARANZ, 2003):

- )Qué fines se desea alcanzar?: Objetivos-competencias

- )Qué deberán aprender los estudiantes?: Contenidos

- )Qué experiencias y procesos de enseñanza-aprendizaje ofrecen mayores posibilidades de alcanzar los objetivos?: Metodología-actividades

- ) Cómo podemos comprobar si se han alcanzado los objetivos o competencias propuestas?: Evaluación

- )Cómo organizar de manera eficaz esas experiencias?: Organización, calendario, temporalización.

De un modo similar Zabalza ${ }^{5}$, en las guías docentes dirigidas a informar y orientar el aprendizaje de los alumnos, distingue las siguientes dimensiones, que describe detenidamente: 1) Datos descriptivos de la materia y de su docencia; 2) Sentido de la materia en el Plan de Estudios; 3) Objetivos de la materia y competencias a desarrollar; 4) Contenidos (teóricos y prácticos) con guía específica para cada tema, incluyendo la bibliografía; 5)Metodología y recursos disponibles, con la distribución de las cargas de trabajo en ECTS; y 6) Evaluación. Por su parte, Mario De Miguel (2006), en un buen trabajo sobre las metologías acordes con el proceso de convergencia de Bolonia, plantea tres dimensiones: 1) Qué pretendemos que aprendan los alumnos (competencias); 2) Cuáles son las modalidades o escenarios y metodologías más adecuadas para que el alumno pueda adquirir estos aprendizajes; y 3) Con qué criterios y procedimientos vamos a comprobar si el alumno las ha adquirido finalmente

La elaboración de Titulaciones debiera adoptar un enfoque curricular. El término “currículum” designa un proyecto formativo integrado (ZABALZA, 2003), donde la planificación de una materia se plantea en relación con el resto del currículum formativo. Como tal, ha de guardar la coherencia interna, que caracteriza al enfoque curricular, en tres componentes:

a) Proyecto: pensado como un conjunto y no como añadido de partes (distintas asignaturas), que ha sido formalizado en un documento, como compromiso público de la Institución.

\footnotetext{
${ }^{5}$ Vid. ZABALZA, Miguel Angel (2005). Guía para la planificación didáctica de la docencia universitaria en el marco del EEES (Guía de guías). Documento disponible en la Asociación Iberoamericana de Didáctica Universitaria. Página Web (Guías docentes): http://www.asoc-aidu.com También específicamente: http://www.arcade.es/aidu/control/files/ 110106111025.doc
} 
b) Formativo: su finalidad es la mejora de la formación de las personas a las que va dirigido, en sus distintas dimensiones y no sólo la enseñanza.

c) Integrado: tiene una coherencia interna y guarda una continuidad, capaz de promover el desarrollo personal y profesional de los estudiantes.

Adoptar un enfoque curricular en el diseño de un Titulación supone superar la mera yuxtaposición de materias para buscar un plan de formación "en el que se ha incluido aquel tipo de experiencias y conocimientos que resultan básicos en relación con el perfil profesional y en el que se ha introducido una secuencia lógica en el proceso que permita optimizar los resultados formativos” (ZABALZA, 2003, p. 24). La perspectiva curricular subraya que las distintas materias deben formar parte, contribuyendo, al proyecto formativo integrado que debiera ser el plan de una carrera. La planificación queda plasmada en la llamada Guía Docente ${ }^{6}$.

Otra nota que caracteriza el enfoque curricular son las dimensiones formativas y educativas, además de las instructivas, como actitudes, ética o relaciones interpersonales. En lugar de dejar “ocultos” dichos aspectos, son planificados en el diseño como experiencias del plan formativo. En este sentido, en las propuestas de Convergencia Europea, dentro de las competencias generales, hay un conjunto de competencias “interpersonales” que apuntan en esta dirección (BOLÍVAR, 2005).

Vamos a describir, sumariamente, algunas notas de cada uno de los elementos de la planificación del programa de la asignatura, de un módulo o unidad didáctica, aún cuando en la práctica deban estar interrelacionados. En particular, será preciso:

- Determinación de los objetivos en términos de competencias

- Una selección y distribución del contenido en unidades didácticas

- Unas estrategias de enseñanza que permitan alcanzar el conocimiento, las actitudes y las habilidades que se han considerado necesarios en la formación inicial de los profesionales.

- Unos sistemas de evaluación que se adapten tanto a la naturaleza de los contenidos a los que se refiere como a las exigencias de aprendizaje.

- Los condicionamientos de calendario o temporalización según número de créditos.

\footnotetext{
${ }^{6}$ La traducción de "Information Package” (término original del ECTS User’s Guide) por “Guía docente” no sería del todo correcta, dentro de nuestro vocabulario didáctico. Sería mejor llamarla "Guía de la Titulación”, reservando "Guía docente” a las orientaciones para la docencia dirigidas a cómo se llevará a cabo la enseñanza de una materia o disciplina; y “Guía didáctica” a las planificaciones o programaciones didácticas de la materia o unidades dirigidas a orientar e informar al alumnado (y eventualmente al profesorado). En cualquier caso, si se conserva el nombre "guía docente”, hay que distinguir distintos niveles de programación: un nivel macro (institución o titulación) y micro (materia), como hace Zabalza (2005). Acceso en 25 jun. 2007
} 


\section{OBJETIVOS (COMPETENCIAS)}

La Universidad tiene la función de formar profesionales capacitados para desempeñar una función social. El diseño de una titulación (y dentro de ella, de modo coherente, de las diversas asignaturas) exige una reflexión para su determinación y concreción, expresados en capacidades o competencias (generales y específicas). Otro asunto, discutible, es que dentro del proceso abierto por la convergencia en un Espacio Europeo de Educación Superior, los contenidos tengan que subordinarse a las competencias del perfil profesional determinado, expresados en objetivos que capaciten para el ejercicio profesional (en el Primer Ciclo) o para la especialización e investigación (Segundo Ciclo o Postgrado).

De acuerdo con los perfiles académicos y profesionales, se entiende, que las competencias representan un factor importante en la formulación de objetivos, que puede guiar la selección de los conocimientos más apropiados para cada objetivo particular. En este nuevo enfoque no sólo importa el qué (contenido) sino el cómo, que hace referencia a la capacitación y modo de aprender del alumno. Así, competencias como trabajo en equipo, desarrollo de las capacidades de síntesis, crítica, pensamiento comparativo, analítico, comunicación y expresión oral y escrita, por citar algunas, son relevantes para el desarrollo personal, académico y profesional de los estudiantes.

El Proyecto Tuning analizó las competencias básicas (y específicas de algunas áreas). Se entiende que las competencias básicas (generales o transversales) deben incluir conocer y comprender (conocimiento teórico de un campo académico, la capacidad de conocerlo y entenderlo), conocer cómo actuar (aplicación práctica y operativa del conocimiento a situaciones determinadas), y conocer cómo ser (valores interpersonales en un contexto social). Junto a las competencias generales, necesarias para actuar profesional, social y cognitivamente en el mundo actual, existen las específicas o más propiamente profesionalizadoras en un área de estudio o asignatura.

\section{CONTENIDOS}

La organización de los contenidos de un campo disciplinar en una propuesta de Diseño o planificación docente se hace atendiendo no sólo a la estructura interna de la materia de que se trata, sino también a las demandas formativas de especialización y formación, y Bsobre todoB a las estrategias didácticas de enseñanza, de forma que posibilite su mejor comprensión y asimilación. Tendríamos así un cierto "trípode”. Se trataría, entonces, de 
relacionar la estructura del contenido de una disciplina escolar (análisis de contenido), con las exigencias formativas o demandas profesionalizadoras (análisis de necesidades), y ambos aspectos implicarán una determinada secuencia de aprendizaje (análisis de tareas).Cuando una Propuesta de acción docente se basa, como a menudo es habitual, en la sola estructura interna de cada disciplina, es más academicista y, en contrapartida, menos innovadora didácticamente.

La introducción del crédito europeo introduce una "ruptura” en la planificación habitual de contenidos de una asignatura. Ahora, se trata de la cantidad de trabajo del alumno (student workload), entendida como el tiempo que el alumno necesita para completar una unidad concreta de enseñanza-aprendizaje. Esto depende de la capacidad del alumno, los métodos de enseñanza-aprendizaje, los recursos didácticos y el diseño de la asignatura. Además, una asignatura en sus contenidos no puede, pues, responder al número de horas de clase presencial, ni tampoco la complejidad o la importancia de una asignatura tiene que ver con los créditos que se le atribuyan. Hay, por tanto, que pasar de un modelo de enseñanza basado en los contenidos a un modelo centrado en el aprendizaje autónomo del alumno, que debe verse reflejado en los programas de enseñanza.

Tres cuestiones principales afectan a los contenidos: Conceptualización (conocimiento de la estructura conceptual y sintáctica del campo disciplinar, y sus posibilidades didácticas), selección (calidad/valor de los contenidos de la enseñanza en conexión con el alumnado al que se dirigen), organización (su mejor estructuración y articulación, para lograr la implicación y comprensión de los estudiantes). Se seleccionan los contenidos que se consideran relevantes para los potenciales estudiantes, de forma que se les proporcionen los fundamentos conceptuales teóricos y los procedimientos o formas de saber hacer que Ben el momento actualB se consideran útiles en la actuación profesional.

\section{METODOLOGÍA- ACTIVIDADES}

Entendemos la metodología como el conjunto de oportunidades y condiciones ofrecidas a los estudiantes, organizadas de manera sistemática e intencional para que promuevan el aprendizaje. Dentro del proceso de convergencia abierto por la Declaración de Bolonia la actividad docente adquiere nuevos enfoques: se pone en primer plano la tutorización y atención más personalizada, organización, seguimiento y evaluación de las actividades no presenciales, coordinación entre actividad presencial y no presencial. De un paradigma centrado en la enseñanza, que ha prevalecido durante muchos años, se pasa a un 
modelo centrado en el aprendizaje del alumno (BARR; TAGG, 1995; ZABALZA, 2004; RUÉ, 2007). El enfoque por competencias tiene, pues, sus propias implicaciones didácticas y representa una oportunidad institucional para la renovación metodológica (DE MIGUEL, 2006). La Comisión para la Renovación de las Metodologías Educativas en la Universidad (CONSEJO DE COORDINACION UNIVERSITARIA, 2006) plantea en sus conclusiones que la reforma de las metodologías educativas se entiende como Aun proceso que es imprescindible abordar para una actualización de la oferta formativa de las universidades españolas@.

En segundo lugar, la competencia docente de un profesor no se mide por el dominio conceptual de los contenidos, aunque sea una condición necesaria; es preciso el empleo de metodologías que provoquen el aprendizaje. “Enseñar Bafirma Zabalza (2002: 123)B es gestionar el proceso completo de enseñanza-aprendizaje que se desarrolla en un contexto determinado, sobre unos contenidos concretos y con un grupo de alumnos con características particulares”. También aquí se impone una cierta reconstrucción de la profesionalidad e identidad del profesorado universitario. Numerosos estudios sobre la enseñanza universitaria han identificado cuatro grandes dimensiones de una enseñanza efectiva: claridad, organización, estimulación del interés y del compromiso de los estudiantes, y un clima positivo en el aula ${ }^{7}$.

De otra parte, la investigación ha puesto de manifiesto que no existe un método óptimo, en términos absolutos, ni un uso universal a prueba de contextos, profesores y contenidos, sino que el método ideal es aquel capaz de ser coherente con la visión de la enseñanza del docente, que se adapta a la identidad, realidad, estilo de aprendizaje y necesidades de cada estudiante y que permita comunicar los contenidos fundamentales. Un equilibrio entre diversos planteamientos metodológicos y una acertada elección en la asignación de las estrategias adoptadas a los objetivos aparejados a los contenidos parece ser el mejor camino. Por eso, se debe tender a una variedad de actividades, ajustada a las circunstancias en que se desarrolle la enseñanza y a las competencias que se pretenden

\footnotetext{
${ }^{7}$ Nira HATIVA, Rachel BARAK y Etty SIMHI. "Exemplary University teachers: Knowledge and beliefs regarding effective teaching dimensions and strategies”, The Journal of Higher Education, v. 72, n. 6, p. 699729, 2001. Una revisión más amplia, el libro de la profesora Nira HATIVA: Teaching for effective learning in Higher Education. Amsterdam: Kluwer, 2001.
} 
promover $^{8}$. A grandes rasgos, las metodologías que se desarrollarán más comúnmente en clase formarán un trípode equilibrado compuesto por a) metodologías expositivas como la lección magistral, b) el aprendizaje autónomo o autoregulado y c) el trabajo en equipo, complementado con la investigación, los proyectos de trabajo, los estudios de caso o el prácticum. Unido a las actividades están los medios o recursos didácticos empleados. Tres medios son útiles en la didáctica universitaria: medios para presentaciones colectivas (retroproyector, presentaciones informatizadas con soporte PowerPoint), medios para apoyar el trabajo en grupo y el trabajo autónomo y medios de apoyo a la tutoría.

El aprendizaje es, pues, un proceso situado, que forma parte y es producto de la propia actividad, contexto y cultura en la que se desarrolla y utiliza (Diaz Barriga, 2003). Por eso mismo,desde esa perspectiva acorde con el enfoque de competencias, aprender (know what) y hacer (know how) son acciones inseparables, por lo que los alumnos deben aprender en el contexto pertinente. Una metodología basada en la resolución de problemas es la más adecuada para el aprendizaje de competencias, ya que busca combinar conocimientos, habilidades, y actitudes en situaciones auténticas o muy cercanas a la realidad. Por ultimo, los materiales y fuentes de información que habitualmente utilizan los estudiantes en su proceso de aprendizaje se organizan en torno a cuatro modalidades: (1) bibliografía; (2) dossiers informativos; (3) carpeta de aprendizaje; (4) material virtual de libre acceso en la red y (5) otras fuentes de información y prácticas.

Si el aprendizaje es, entonces, un proceso constructivo, más que reproductivo, y es un proceso social, cultural e interpersonal gobernado por factores sociales y situacionales tanto como por los cognitivos; entonces las estrategias didácticas del aula también deben cambiar de enfoque. La enseñanza es vista ahora más como una tarea de orquestar un complejo entorno de aprendizaje y actividades, en lugar de una "línea de montaje” donde el conocimiento sea transferido de alguien que conoce a individuos que no saben, mediante un monólogo. El profesor actúa de guía, facilitador y organizador de un entorno adecuado para el proceso de aprendizaje de los estudiantes.

\footnotetext{
${ }^{8}$ Se puede ver disponibles en Internet, entre otros, Fernández March, Amparo: “Metodologías activas para la formación por competencias”. Educatio XXI, 24, pp. 35-56. Disponible en http://www.um.es/educatio Igualmente la investigación coordinada por Mario de Miguel (posteriormente publicada en libro, DE MIGUEL, 2006): Modalidades de enseñanza centradas en el desarrollo de las competencias. Madrid: Ministerio de Educación y Ciencia, 2005. Programa Estudios y Análisis. Disponible en Internet: URL .http://www.mec.es/univ/proyectos2005/EA2005-0118.pdf Acceso en 8 abr. 2007.
} 
$\mathrm{Al}$ respecto una nueva agenda sobre la calidad de la enseñanza y el aprendizaje en la Universidad, a nivel internacional, se centra, desde el Report Boyer (1990) de la Carnegie Foundation, en el marco del "the scholarship of teaching”. Este enfoque permite situar debidamente (SHULMAN, 2004) dentro del trabajo académico (y no sólo como un asunto pedagógico o didáctico) que la enseñanza está “centrada en el aprendizaje del estudiante, como se reclama en el EEES. Al igual que la investigación, la enseñanza ha de llegar a hacerse visible o pública, como una Apropiedad comunitaria” (“community property”, lo llama Shulman, 2004). Además de este carácter, ha de ser sometida al análisis y comentario de una apropiada comunidad de compañeros, en analogía con los procesos de revisión por pares y calidad de los productos de la investigación. De este modo, una comunidad académica, que está comprometida con su trabajo académico, trata su trabajo como un acontecimiento público, sometido al escrutinio de pares. En tercer lugar, el trabajo académico docente ha de ser intercambiado, de manera que otros tengan posibilidad de aprender ${ }^{9}$.

\section{EVALUACIÓN}

Por su parte, el papel de la evaluación ha de cambiar, con motivo de la integración en el Espacio Europeo de Educación Superior, tanto porque ha de valorar el trabajo total como porque ha de dirigirse a las competencias adquiridas. Se entienden éstas como capacidades de movilizar recursos (saberes, capacidades, informaciones, etc.) para actuar con pertinencia y eficacia en un conjunto de situaciones, que se ponen de manifiesto obrando en una actividad compleja, con un determinado grado de maestría (Villardón, 2006). En este sentido, significan la aplicación, de manera efectiva, de conocimiento utilizable y habilidades en un contexto específico.

Además, el cambio en el énfasis hace que la evaluación del alumno pase del conocimiento como referencia dominante (incluso la única referencia) a incluir una valoración centrada en las capacidades, destrezas y procedimientos relacionados con el trabajo y la actividad afines al desarrollo del alumno y en relación con los perfiles académicos y profesionales ya definidos, lo que también muestra una mayor variedad de estrategias de

\footnotetext{
${ }^{9}$ Se puede ver la página web informativa del Proyecto de Investigación que dirigimos: http://www.ugr.es/ proexc. Contiene una exhaustiva bibliografía sobre el tema. Además se han recogido, a nivel mundial, los recursos disponibles (Bibliografías, proyectos similares, revistas, otras publicaciones, videos), así como Centros de Investigación sobre la Enseñanza universitaria.
} 
valoración. Una nueva conceptualización y práctica de la evaluación en la enseñanza universitaria, sin duda, pasa por (Bordás y Cabrera, 2001) cuatro ejes:

a) Superar la concepción de una evaluación puntual, al final de cuatrimestre o curso

b) Inclusión de la evaluación continua o formativa

c) Ampliación de los objetivos de la evaluación de los aprendizajes

d) Inclusión de la evaluación compartida

El llamado “portafolios”, que preferimos traducir por “carpeta de aprendizaje”, en los últimos años -desde el contexto anglosajón- se ha ido convirtiendo en una Anueva ortodoxia@ en la evaluación del aprendizaje universitario (LYONS, 1999). A ello se une que es especialmente adecuado en la nueva perspectiva del crédito europeo (ECTS), como medio para dar cuenta (de-mostrar) credencialmente de los trabajos realizados y de las competencias. De modo sistemático y organizado se documenta, como una cierta antología secuencial, todo aquello que contribuya a mostrar o ejemplificar el progreso del conocimiento adquirido en un campo o materia, así como la reflexión autoevaluativa del trabajo realizado.

\section{EL CRÉDITO EUROPEO: PAPEL, SIGNIFICADO Y DISTRIBUCIÓN}

El llamado “crédito europeo” o ECTS en siglas, a diferencia de los modos tradicionales, no es una medida de duración temporal de las clases impartidas por el profesor, sino una unidad de valoración del volumen de trabajo total del alumno, expresado en horas, que incluye tanto las clases, teóricas o prácticas, como el esfuerzo dedicado al estudio, a la realización de trabajos, proyectos y lectura de documentos, así como a la preparación y realización de exámenes. Representa, pues, como unidad de medida del trabajo académico, la cantidad de trabajo del estudiante para cumplir los objetivos del programa de estudios.

De este modo, el crédito europeo mide la cantidad de trabajo medio del alumno, entendida como el tiempo que éste necesita para dominar una unidad de enseñanzaaprendizaje $^{10}$. Mientras los créditos actuales son unidades de acumulación, que tienen en cuenta exclusivamente las horas lectivas, los créditos ECTS representan los valores numéricos asignados a cada materia o asignatura para describir el trabajo necesario (student workload) que un estudiante debe realizar para prepararla y superarla. En los créditos se incluyen clases teóricas, clases prácticas, seminarios, tutorías, trabajos de campo, horas de estudio, exámenes

\footnotetext{
${ }^{10}$ Página de la Comisión Europea (Education and Training) sobre ECTS. Users' Guide and Forms http://ec.europa.eu/education/programmes/socrates/ects/guide_en.html. Documento de la ANECA sobre el crédito europeo. Disponible en Internet http://www.aneca.es/publicaciones/docs/publi_credito\%20europeo.pdf.
} 
y otro tipo de evaluaciones, etc. Igualmente, los créditos se podrán conseguir también fuera de las instituciones de educación superior, incluyendo la experiencia adquirida a lo largo de la vida (lifelong learning), siempre que esté reconocida por las universidades receptoras.

El crédito europeo responde a un enfoque centrado en el alumno más que en el profesor. Existe una clara relación entre las estructuras educativas, los resultados de aprendizaje, la cantidad de trabajo del alumno y el cálculo de créditos en particular, dentro del contexto del proceso de Bolonia. Estos elementos son muy importantes hoy día en que la enseñanza tradicional está siendo sustituida parcialmente por nuevos tipos de enseñanza y aprendizaje. Éste es, sin duda, el principal desafío pedagógico al que se enfrentan las universidades europeas en los próximos años. La transición, de un modelo de enseñanza tradicional a un modelo centrado en el aprendizaje autónomo del alumno.

El trabajo relativo del estudiante (relative student workload) es difícil de definir con exactitud, pues siempre será un modo de estimar la carga de trabajo. Los créditos dependen entonces de la cantidad de tiempo que le lleva a un alumno medio aprender la asignatura o completar el curso de manera exitosa. En una cultura universitaria como la española en la que el peso actual de un curso se estima en relación con el esfuerzo del profesor, hay dificultades para calcular créditos para cursos basados en el esfuerzo del estudiante. En cualquier caso, tampoco el asunto se cifra, como ha sucedido en algunas experiencias de reforma, en calcular de modo minucioso los tiempos escolares, en una "taylorización" de los tiempos requeridos, como ha criticado Gimeno (2005).

\section{PLANIFICAR POR COMPETENCIAS. UN ANÁLISIS CRÍTICO}

El debate sobre el nuevo diseño de las titulaciones en términos de competencias no es sólo técnico, primariamente es político e ideológico: cuál debe ser la función de la Universidad en relación con la formación (formar para empleo o introducir en un ámbito cultural) y el tipo de profesionalidad requerida. El discurso de las "competencias" supone redefinir la profesionalidad como la regulación de un listado de competencias para la enseñanza, que los estudiantes han de adquirir para conseguir el título. En un plano general, lo que se discute es el mismo modelo de Universidad, que debe mediar entre modelo académico (Humboldt) y el profesionalizador (anglosajón o neoliberal). Una herencia de la educación liberal es que la enseñanza universitaria ha de proporcionar una formación de la inteligencia y el saber crítico, conjugada con un saber especializado profesionalizador. 
Una competencia se caracteriza por la capacidad de movilizar recursos (saberes, capacidades, informaciones, etc.) para actuar con pertinencia y eficacia en un conjunto de situaciones, dando lugar al resultado deseado. Se pone de manifiesto actuando en una actividad compleja con un determinado grado de dominio o maestría (PERRENOUD, 2001). En este sentido significa la aplicación de manera efectiva de conocimiento utilizable y habilidades en un contexto específico. Más específicamente, el concepto de competencia tendría en educación dos denotaciones: desde una perspectiva teórica, competencia es concebida como una estructura cognitiva que facilita conductas específicas. Desde una perspectiva operativa, una competencia parece cubrir un amplio espectro de habilidades para funcionar en situaciones complejas, lo que supone conocimiento, actitudes, pensamiento metacognitivo y estratégico. Las competencias tienen, pues, un componente mental de pensamiento representacional y otro conductual mediante el componente de actuación.

Una competencia profesional comprende, al menos, cuatro aspectos: integra, moviliza u orquesta recursos; dicha movilización es pertinente con las situaciones, suponen operaciones mentales para determinar acciones pertinentes a las situaciones, y se construyen tanto en la formación inicial como en el propio ejercicio profesional. Si la competencia se apoya en el conocimiento, no se reduce -sin embargo- a él. Como "gramática generadora de prácticas”, más que un saber dado, integran u orquesta determinados recursos, que son pertinentes en una determinada situación, y se construyen tanto en la formación como en la navegación cotidiana de la práctica.

El Proyecto Tuning entiende "competencia”, como "una combinación de atributos que describen el nivel o grado de suficiencia con que una persona es capaz de desempeñarlos” (GONZÁLEZ; WAGENAAR, 2003, p. 80). Posteriormente, especifica que es “una combinación dinámica de conocimiento, compresión, capacidades y habilidades” (GONZÁLEZ; WAGENAAR, 2005, p. 379). Entre estos atributos están conocimientos conceptuales (conocer y comprender), saber cómo actuar (aplicación del conocimiento a situaciones por medio de destrezas o habilidades) y saber cómo ser (valores y compromiso ético). Las competencias pueden ser verificadas y evaluadas en función del grado de desempeño. Además de distinguir competencias específicas (vinculadas a áreas de estudio de una Titulación) y competencias genéricas (comunes a cualquier titulación), clasifica estas últimas, de un modo discutible, en instrumentales, interpersonales y sistémicas. No se pueden establecer un “ranking” de competencias, a partir de cuestionarios a empleadores, graduados y académicos. 
Como apunté en otro lugar (BOLÍVAR, 2006), en el caso español, es necesaria una crítica a las limitaciones del Modelo de Diseño adoptado ((Modelo Tuning) en el particular maridaje establecido entre este Proyecto y la ANECA. La metodología inductiva empleada en la selección de competencias de cada perfil profesional (GONZÁLEZ; WAGENAAR, 2003) se realiza por un cuestionario a tres grupos (empleadores, graduados y académicos), lo que conduce a establecer un "ranking” de competencias, al tiempo que una discutible subordinación a los llamados “empleadores”. Como establece Frank Weiner (2004) en un excelente trabajo sobre la conceptualización de competencia, el punto de vista ha de ser un enfoque normativo con un marco teórico consolidado y no un punto de partida empírico, que impediría ir más allá de la adaptación del individuo al mundo actual (p. 107). El concepto de competencia es, sin embargo, plural, con varios enfoques en su configuración (DELAMARE; WINTERTON, 2005), que una perspectiva académica debiera tener presentes, para adoptar una perspectiva más holística. Un modelo de competencias no puede ser inductivo (a partir de las encuestas), pues ello da lugar siempre a una debilidad de conceptualización y la clasificación de competencias establecida será contingente, dependiente del contexto.

El Proyecto DeSeCo ${ }^{11}$, como la conceptualización más potente sobre las competencias, constituye una buena base para establecer un discurso coherente sobre el tema (BOLÍVAR; PEREYRA, 2006). Sin embargo se dirige a la educación obligatoria, no a la universitaria, como muestran las competencias clave necesarias para la vida (interactuar en grupos socialmente heterogéneos, actuar con autonomía y utilizar herramientas interactivamente). En cualquier caso, tiene la especificidad de -por una parte- hacer una fundamentación interdiciplinar de las competencias; por otra, expandirlas del marco escolar, al que hasta entonces se habían ceñido las evaluaciones, considerando que limitarse a las competencias con base en las materias del currículum escolar es insuficiente individual y socialmente, si no se completan con otras competencias relevantes para la vida (realización individual y participación efectiva en la sociedad).

DeSeCo aboga por un modelo holístico de competencia (RYCHEN; SALGANIK, 2006, p. 73-90), que integra y relaciona las demandas, los prerrequisitos cognitivos y no

\footnotetext{
${ }^{11}$ Sobre el Proyecto DeSeCo (Definition and selection of key competencies), auspiciado por la OCDE y coordinado por Dominique S. Rychen y Laura H. Salganik, se puede ver, de entrada, el "Executive Summary” en la web del Proyecto http://www.deseco.admin.ch , así como los restantes documentos incluidos en dicha web. En español contamos con la edición del Primer Informe (2001) en: RYCHEN, D.S.; SALGANIK, L.H. (eds.).

Definir y seleccionar las competencias fundamentales para la vida. Trad. de Leticia O. García Cortés. México: Fondo de Cultura Económica, 2004. El Informe Final por su parte ha sido editado por Antonio Bolívar y Miguel A. Pereyra con traducción de J. M. Pomares (RYCHEN; SALGANIK, 2006).
} 
cognitivos y el contexto, en un complejo sistema de acción. Como constructo social, DeSeCo apuesta por una conceptualización que sea científicamente plausible y pragmáticamente relevante. Una competencia se define “como la habilidad para satisfacer con éxito exigencias complejas en un contexto determinado, mediante la movilización de prerrequisitos psicosociales que incluyen aspectos tanto cognitivos como no cognitivos” (RYCHEN; SALGANIK, 2006, p. 74). Un enfoque funcional, orientado a la demanda, sitúa en el centro los resultados obtenidos por el individuo mediante una acción determinada, al tiempo que, como contextualmente dependiente, no existan independientemente del contexto y de la acción.

En el modelo de planificación por competencias, tipo Tuning, al tener que especificar o desagregar las competencias generales, transversales o complejas en tareas más simples o competencias derivadas, necesariamente se suele abocar a comportamientos o competencias simples, especificando incluso niveles de ejecución deseables, "que suele coincidir con la formulación de objetivos específicos”, como señala Diaz Barriga (2006, p. 31). Esto hace que, al final, el enfoque por competencias coincida (o nos haga regresar) a la "pedagogía por objetivos” de los sesenta del siglo anterior. Al respecto comenta el autor: "No se percibe pero la estrategia de implementación de un objetivo conductual es la misma que subyace en la que se sigue para descomponer una competencia compleja en una derivada o simple y al mismo tiempo establecer en ésta un nivel de ejecución” (p. 31). Sin quererlo, al final, el enfoque por competencias aboca a una planificación por objetivos de aprendizaje, ahora "reformateados" en términos de competencias específicas o simples. De hecho, los resultados del aprendizaje (learning outcomes) se entienden criterios de evaluación de lo que se espera que consigan los estudiantes al final de un período de trabajo, formulados en términos de competencias (GONZÁLEZ; WAGENAAR, 2005, p. 162-383).

Partir del perfil profesional, como base para la toma de decisiones siguientes, supone subordinar la enseñanza universitaria al mundo laboral "empleadores”, expresado ahora en términos de competencias. Como no ocultan sus mentores, en la sociedad globalizada, se quiere contar con un mercado laboral flexible: formar individuos que posean activos competenciales para adaptarse a un futuro laboral cambiante, en un aprendizaje a lo largo de la vida. Su procedencia del mundo empresarial y profesional lo hacen sospechoso al vincularlo a las políticas neoliberales que subordinan la educación a las demandas del mercado, como ha criticado Boaventura de Sousa (2005) desde una perspectiva crítica y general. 
La “metodología Tuning”, adoptada acríticamente en España por la ANECA en el nuevo diseño de las Titulaciones, como un cierto “pensamiento único”, sin hacerse eco siquiera de otras perspectivas más amplias, ha sido objeto -con razón- de análisis críticos (MARTÍNEZ BONAFÉ, 2005), en especial por romper con un modelo de Educación Superior humboltiano, al tiempo que supone una subordinación a las demandas del mercado, de acuerdo con las políticas neoliberales. En unos casos, las competencias “transversales” son obvias; las "específicas” en exceso particulares y reguladoras, hasta el extremo de querer ponderar el peso (y el tiempo) de cada competencia en cada contenido. Tal como se está empleando supone “reformatear” los programas en términos de una suma de competencias terminales, recordando las estrategias propias de los años setenta de "programación por objetivos”, al subordinar todo el proceso didáctico a los objetivos, que los alumnos deberán ser capaces de dominar al final de un proceso educativo.

Al final, la tarea formativa universitaria queda limitada a unos listados de competencias que recuerdan a los elaborados para formar, seleccionar y reclutar recursos humanos en el mundo de la empresa. Centrar la enseñanza en el aprendizaje de competencias profesionales, a la larga (como, de hecho, está pasando en USA) puede llevar a "certificar aprendizajes”, que puede hacer también otras instituciones (universidades virtuales, privadas, exámenes externos, etc.). La enseñanza universitaria es vista como una colección de habilidades que pueden ser analizadas, descritas y entrenadas. Como se afirmaba en un Manifiesto de Profesores e investigadores universitarios ${ }^{12}$ : "Nos preocupa que, con el argumento de que la universidad debe atender a las demandas sociales, haciendo una interpretación claramente reduccionista de qué sea la sociedad, en realidad se ponga a la universidad al exclusivo servicio de la empresas y se atienda únicamente a la formación de los profesionales solicitados por éstas”.

Sin ser las competencias ni “angel” ni “demonio”, hemos de ser conscientes de las graves limitaciones, aparte de las virtualidades que también puedan tener. Las llamadas “competencias genéricas”, en unos casos son obvias, en otros ya se debían poseer, finalmente debían ser conjuntas de todas las asignaturas. Y es que un enfoque de competencias requiere superar las lógicas disciplinares por planteamientos más transversales o interdisciplinares. En segundo lugar, más relevante, es que en cualquier caso, si es que haya de pasar de un enfoque

\footnotetext{
${ }^{12}$ ¿Qué Educación Superior Europea? (marzo 2005). Se puede ver el “Debate sobre la Convergencia Europea” en la Web: http://fs morente.filos.ucm.es/debate/inicio.htm.
} 
centrado en la enseñanza a centrarlo en el aprendizaje (un principio, por lo demás, obvio en cualquier metodología didáctica), se ha de empezar por promover el reconocimiento y valoración de la calidad docente, conjugada con la investigación. La mejora de la docencia pasa por una valoración similar a la investigación. El referido enfoque del "scholarship of teaching and learning”, proporciona una buena plataforma al respecto.

Es verdad, no obstante, como señala Ronald Barnett (2001, p.105), que "los nuevos vocabularios no caen del cielo, sino que surgen orgánicamente de las actividades colectivas y las reflejan”. En caso de la Formación Profesional y Educación Superior, formular los estudios en términos de perfiles profesionales, que den lugar a las correspondientes competencias, es expresión de nuevos modos de regulación e intervención del mundo empresarial y estatal que, en función de una racionalidad instrumental, exige pasar la enseñanza de un proceso a un producto. Como el propio Barnett dice a continuación:

En principio, no puede existir objeción para el uso de estos términos (competencia y resultados) en el contexto del proceso educativo. Sin embargo, si caracterizamos los procesos educativos primordialmente en estos términos y los utilizamos como criterios para diseñarlos y evaluarlos, podemos entrar en un terreno preocupante (BARNETT, 2001, p.108).

La educación superior, aún en aquellas carreras que tienen perfiles profesionales claros, deber tener unos objetivos más amplios que las competencias profesionales. Además, no puede limitarse a actuaciones exitosas en situaciones predecibles, debe ir más lejos, planteando posibles respuestas innovadoras o creativas en situaciones no predecibles. Con el discurso de las competencias se pierden otros vocabularios relevantes en la enseñanza: la comprensión, la crítica, la interdisciplinariedad y la sabiduría, entre otros.

\section{PARA CONCLUIR}

La reforma de Bolonia tiene una doble dimensión: armonizar las estructuras, de modo que posibilite la movilidad y el acceso al mercado laboral entre todos los países europeos. Al tiempo, se pretende incrementar la calidad mediante mecanismos de evaluación externos y de la competitividad entre las instituciones universitarias. Además, está la dimensión cualitativa de mejora de la enseñanza y el aprendizaje, uno de cuyos primeros elementos es una buena planificación, a nivel institucional (Titulación) y de cada materia (Departamento y enseñanza). La planificación por competencias pretende vincular la mejora de la enseñanza con el mercado laboral, al subordinarse a determinados perfiles profesionales, determinados por el mercado. Esta segunda dimensión es la que hemos analizado críticamente: si bien la 
Universidad debe preparar para el ejercicio profesional, ello no puede suponer desdeñar otras dimensiones de la educación superior.

El enfoque por competencias, por sí mismo, no conlleva un modelo de planificación o diseño curricular. La determinación de competencias genéricas o profesionales puede, efectivamente, contribuir a delimitar las tareas que deben dominar los estudiantes; pero, como señala Diaz Barriga (2006), “no permite una construcción curricular consistente, no permite orientar con precisión la elaboración de un plan de estudios ni la forma de graduar el trabajo en su interior” (DIAZ BARRIGA, 2006, p. 34-35). Más puede contribuir, en una perspectiva abierta, a un cambio metodológico y en los procedimientos de la evaluación, a condición de que la planificación no quede sólo como un documento burocrático, elaborado como respuesta a requerimiento administrativo.

La reforma en curso amenaza con quedarse en una reforma estructural más, sin llegar a la mejora de los modos de enseñar y aprender. El asunto, en último extremo, es cómo incidir en la cultura o "gramática básica” de la enseñanza universitaria, es decir en los modos mismos de enseñar y aprender, para que no quede como un mero "reformateo" de los títulos y asignaturas, como denunciabamos en otro lugar (BOLÍVAR, 2006), con motivo del nuevo diseño de los planes universitarios de formación de maestros. Los cambios de “cultura” suelen requerir, para no quedarse en prédicas retóricas, cambios organizativos que supongan otras formas de hacer de profesores y alumnos. Si no queremos conformarnos con cómo la gente actúa en una organización, se requieren otros roles y estructuras que apoyen y promuevan las prácticas educativas que deseamos. El Comisión para la Renovación de las Metodologías Educativas en la Universidad, entre otros, reclamaba:

\footnotetext{
La adecuación al futuro Espacio Europeo de Educación Superior requiere abordar un proceso interuniversitario, institucional y estructurado -algo que no se percibe en este momento- de renovación pedagógica, que contemple sucesivas fases: impulso (información, sensibilización, motivación, diseño de planes); formación del profesorado; ejecución (proyectos piloto, elaboración de guías y materiales, constitución de redes y canales para el intercambio de experiencias); difusión y evaluación (catálogo de buenas prácticas -no sólo nacionales-, congresos, seminarios, publicaciones). En paralelo, habrá que atender otras cuestiones, como, por ejemplo, la adecuación de los mecanismos de financiación o las infraestructuras. (CONSEJO DE COORDINACIÓN UNIVERSITARIA, 2006, p. 135).
}

No obstante, la reforma de los planes de estudio universitarios tiene la virtualidad de que los diversos equipos docentes se replanteen el sentido de la enseñanza universitaria, dando ahora prioridad a un aprendizaje funcional y relevante para el posterior ejercicio profesional. Romper con el individualismo dominante en la docencia universitaria, heredero 
de una larga tradición liberal que entiende la profesionalidad como autonomía, para dar lugar a un trabajo conjunto, es un vía prometedora de mejora docente. Esta se potenciada cuando los compañeros, como colegas, además de compartir espacios y ámbitos comunes de trabajo, intercambian experiencias y colaboran sobre los modos más adecuados de enseñar y promover el aprendizaje de los estudiantes.

\section{REFERENCIAS}

BARNETT, R. Los límites de la competencia. El conocimiento, la educación superior y la sociedad. Barcelona: Gedisa, 2001. 286 p.

BARR, R.; TAGG, J. From teaching to learning: a new paradigm for undergraduate education. Change, v. 27, n.6, p. 13-25, nov/dec. 1995.

BOLÍVAR, A. El lugar de la ética profesional en la formación universitaria. Revista Mexicana de Investigación Educativa, v.10, n. 24, p. 93-123, ene/mar. 2005. Disponible en Internet: http://www.cesu.unam.mx/rmie/num24/24-ensayo.pdf. Acceso en: 23 fev. 2006

. La formación inicial del profesorado y el desarrollo de las instituciones de formación. In: ESCUDERO, J. M.; LUIS, A. (Eds.). La mejora de la educación y la formación del profesorado. Políticas y prácticas. Barcelona: Octaedro, 2006. p. 123-154.

.; PEREYRA, M. A. El proyecto DeSeCo sobre la definición y selección de competencias clave. Introducción a la edición española. In: RYCHEN, D. S.; SALGANIK L. H. (Eds.). Las competencias clave para el bienestar personal, social y económico. Archidona (Málaga): Ediciones Aljibe, 2006. p. 1-13.

BORDÁS, I.; CABRERA, F. A. L'avaluació de l'alumnat a la universitat. Educar, n. 28, p. 61-82, 2001. Disponible en Internet: http://ddd.uab.es/pub/educar/0211819Xn28p61.pdf Acceso en: 24 jun. 2007.

BOYER, E. Scholarship reconsidered: priorities of the professoriate. Princeton, N.J.: The Carnegie Foundation for the Advancement of Teaching. 1990.147 p.

COLÁS, P.; DE PABLOS, J. (Eds.). La universidad en la unión europea. El espacio europeo de educación superior y su impacto en la docencia. Archidona (Málaga): Aljibe, 2005. $201 \mathrm{p}$.

COMISIÓN EUROPEA. Las competencias clave. Un concepto en expansión dentro de la educación general obligatoria. Bruxelas: Eurydice, 2003. 186 p. Disponible en Internet: http://www.eurydice.org/ressources/eurydice/pdf/0_integral/032ES.pdf Acceso en: 24 jun. 2007.

CONSEJO DE COORDINACIÓN UNIVERSITARIA. Propuestas metodológicas para la renovación de las metodologías educativas. Madrid: Ministerio de Educación y Ciencia, 2006. 286 p. Disponible en Internet: http://www.mec.es/educa/ccuniv/html/metodologias/ Acceso en: 14 jun. 2007. 
COROMINAS, E. Competencias genéricas en la formación universitaria. Revista de Educación, n. 325, p. 299-331, 2001.

DELAMARE LE DEIS, F.; WINTERTON, J. What is competence? Human Resource Development International, v. 8, n. 1, p. 27-46, 2005.

DE MIGUEL, M. (Dir.). Metodología de enseñanzas y aprendizaje para el desarrollo de competencias: orientaciones para el profesorado universitario ante el Espacio Europeo de Educación Superior. Madrid: Alianza Editorial, 2006. 232 p.

DIAZ BARRIGA, A. El enfoque de competencias en educación. ¿Una alternativa o un disfraz de cambio? Perfiles educativos, v. 28, n. 111, p. 7-36, 2006. Disponible en Internet: http://redalyc.uaemex.mx/. Acceso en: 14 jun. 2007.

ENQA (European Association For Quality Assurance in Higher Education) (2005). Standars and guidelines for Quality Assurance in the European Higher Education Area. Helsinki: ENQA. Disponible en Internet: http://www.bologna-bergen2005.no/Docs/ 00-Main doc/ 050221_ENQA_report.pdf Acceso en: 14 mai. 2007

ESTEBARANZ, A. La planificación en la universidad. In: MAYOR, C. (Coord.). Enseñanza y aprendizaje en la educación superior. Barcelona: Octaedro-EUB, 2003. p. 83-111.

GIMENO, J. Nos habíamos olvidado del alma mater. ¿Innovación en la universidad con motivo de la convergencia europea de las universidades? In: GIMENO, J. La educación que aún es posible. Madrid: Morata, 2005, p. 160-180.

GONZÁLEZ, J.; WAGENAAR, R. Tuning educational structures in Europe. I. Final report. Bilbao: Universidad de Deusto, 2003. 316 p. Disponible en Internet:

http://tuning.unideusto.org/tuningeu/. Acceso en: 12 fev. 2007.

GONZÁLEZ, J.; WAGENAAR, R. G. (Eds.) Tuning educational structures in Europe. II. Universities' contribution to the Bologna Process. Bilbao: Publicaciones de la Universidad de Deusto, 2005. 386 p. Disponible en Internet: http://www.tuning.unideusto.org/. Acceso en: 12 fev. 2007.

GOÑI ZABALA, J. M. El espacio europeo de educación superior, un reto para la universidad: competencias, tareas y evaluación, los ejes del currículum universitario. Barcelona: Octaedro, 2005. 175 p.

LYONS, N. El uso de portafolios. Propuestas para un nuevo profesionalismo docente. Buenos Aires: Amorrortu, 1999. 351 p.

MARTÍNEZ BONAFE, J. La formación del profesorado y el discurso de las competencias. Revista Interuniversitaria de Formación del Profesorado, v.18, n.3, p.127-144, 2005.

PEREYRA, M. A.; LUZÓN, A.; SEVILLA, D. Las universidades españolas y el proceso de construcción del Espacio Europeo de Educación Superior. Limitaciones y perspectivas de cambio. Revista Española de Educación Comparada, v. 12, p. 113-143, 2006. 
PERRENOUD, P. Porquê construir competências a partir da escola? Desenvolvimento da autonomia e luta contra as desigualdades. Porto: Edições ASA, 2001. 128 p.

La universitat entre la transmissió de coneixements i el desenvolupament de competències. Quaderns de docència universitària, n. 5, p. 27-52, 2006. (Institut de Ciències de l'Educació de la Universitat de Barcelona). Disponible en Internet: http://www.unige.ch/fapse/SSE/teachers/perrenoud/php_main/php_2004/2004_07.html.

RUÉ, J. Enseñar en la Universidad. El EEES como reto para la educación superior. Madrid: Narcea, 2007. 224 p.

RYCHEN, D. S.; SALGANIK L. H. (Eds.). Las competencias clave para el bienestar personal, social y económico. Archidona (Málaga): Ediciones Aljibe, 2006. 210 p.

SHULMAN, L. S. Teaching as community property: essays on higher education. San Francisco: Jossey-Bass, (2004). 272 p.

SOUSA SANTOS, B. La universidad en el siglo XXI. Para una reforma democrática y emancipadora de la universidad. Buenos Aires: Laboratorio de Políticas Públicas y Ed. Miño y Dávila, 2005. 88 p.

VILLARDÓN, L. Evaluación del aprendizaje para promover el desarrollo de las competencias. Educatio XXI, n. 24, p.57-76, 2006. Disponible en Internet:

$<$ http//www.um.es/educatio $>$ Acceso en: 25 jun. 2007

WEINER, F. E. Concepto de competencia: una aclaración conceptual. In: RYCHEN, D. S.; SALGANIK, L. H. (Eds.). Definir y seleccionar las competencias fundamentales para la vida. Mexico: Fondo de Cultura Economica, p. 94-127, 2004.

YÁNIZ, Concepción. Planificar la enseñanza universitaria para el desarrollo de las competencias. Educatio XXI, n. 24, p. 17-34, 2006. Disponible en Internet:

$<$ http//www.um.es/educatio> Acceso en: 25 jun. 2007

ZABALZA, M. A. La enseñanza universitaria: el escenario y los protagonistas. Madrid: Narcea, 2002. 238 p.

Competencias docentes del profesorado universitario. Calidad y desarrollo profesional. Madrid: Narcea, 2003. 253 p.

A Didáctica Universitaria. Un espazo disciplinar para estudo e mellora da nosa docencia. Lección inaugural del curso académico 2004-5. Universidad de Santiago de Compostela, 2005. 67 p. Disponible en Internet:

http://www.usc.es/intro/doc/discurso_inaugural.pdf. Acceso en: 25 jun. 2007. 
ANTONIO BOLÍVAR

Doctor en Filosofía y Ciencias de la Educación Catedrático de Didáctica y Organización Escolar

Facultad de Ciencias de la Educación Universidad de Granada (España)

E-mail: abolivar@ugr.es

Recebido em: 22/11/2007

Publicado em: 18/01/2008 